TITLE:

\title{
Intrafractional tracking accuracy in infrared marker-based hybrid dynamic tumour-tracking irradiation with a gimballed linac.
}

\section{AUTHOR(S):}

Mukumoto, Nobutaka; Nakamura, Mitsuhiro; Yamada, Masahiro; Takahashi, Kunio; Tanabe, Hiroaki; Yano, Shinsuke; Miyabe, Yuki; ... Sawada, Akira; Kokubo, Masaki; Hiraoka, Masahiro

\section{CITATION:}

Mukumoto, Nobutaka ... [et al]. Intrafractional tracking accuracy in infrared marker-based hybrid dynamic tumourtracking irradiation with a gimballed linac.. Radiotherapy and oncology : journal of the European Society for Therapeutic Radiology and Oncology 2014, 111(2): 301-305

\section{ISSUE DATE:}

2014-05

URL:

http://hdl.handle.net/2433/188903

\section{RIGHT:}

(c) 2014 Elsevier Ireland Ltd.; This is not the published version. Please cite only the published version.; この論文は出版社版でありません。引 用の際には出版社版をご確認ご利用ください。 
1 Intrafractional tracking accuracy in infrared marker-based hybrid

2 dynamic tumour-tracking irradiation with a gimballed linac

4 Nobutaka Mukumoto ${ }^{\text {a }}$, Mitsuhiro Nakamura ${ }^{\text {a,*, }}$, Masahiro Yamada ${ }^{\text {a }}$ Kunio

5 Takahashi $^{\mathrm{b}}$, Hiroaki Tanabe ${ }^{\mathrm{c}}$, Shinsuke Yano $^{\mathrm{d}}$, Yuki Miyabe ${ }^{\mathrm{a}}$, Nami Ueki ${ }^{\mathrm{a}}$, Shuji

6 Kaneko $^{\text {a }}$, Yukinori Matsuo ${ }^{\text {a }}$, Takashi Mizowaki ${ }^{\mathrm{a}}$, Akira Sawada ${ }^{\mathrm{a}, \mathrm{e}}$, Masaki Kokubo ${ }^{\mathrm{c}, \mathrm{f}}$,

7 and Masahiro Hiraoka ${ }^{a}$

$8{ }^{\text {a }}$ Department of Radiation Oncology and Image-applied Therapy, Graduate School of

9 Medicine, Kyoto University, Kyoto, Japan

10 b Advanced Mechanical Systems Department, Mitsubishi Heavy Industries Ltd, Hiroshima,

11 Japan

$12{ }^{\mathbf{c}}$ Division of Radiation Oncology, Institute of Biomedical Research and Innovation, Hyogo,

13 Japan

$14{ }^{\mathbf{d}}$ Division of Clinical Radiology Service, Kyoto University Hospital, Kyoto, Japan

$15{ }^{\mathrm{e}}$ Department of Radiological Technology, Faculty of Medical Science, Kyoto College of

16 Medical Science, Kyoto, Japan

$17{ }^{\mathbf{f}}$ Department of Radiation Oncology, Kobe City Medical Center General Hospital, Hyogo, 18 Japan

20 * Corresponding author: Mitsuhiro Nakamura, Ph.D., Graduate School of Medicine,

21 Kyoto University, 54 Kawahara-cho, Shogoin, Sakyo-ku, Kyoto, 606-8507, Japan.

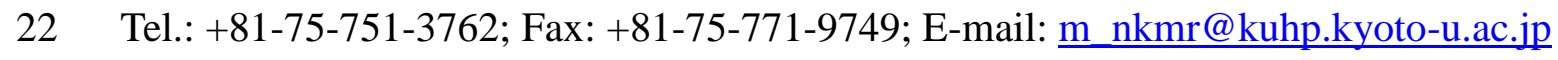


24 Total number of pages: 16 (Tables: 2; Figures: 2; Supplementary Material: 1)

25

26 Running title: Intrafractional accuracy of IR Tracking

27

28 Key words: Four-dimensional image-guided radiotherapy, dynamic tumour-tracking 29 irradiation, respiratory motion, Vero4DRT, intra-fractional tracking accuracy.

30

31 Meeting presentation: This work was accepted for presentation during the poster viewing

32 session of the $54^{\text {th }}$ Annual Meeting of the American Society for Radiation Oncology in

33 Boston, October 28-31, 2012.

34 


\section{Intrafractional accuracy of IR Tracking}

\section{ABSTRACT}

36 Purpose: To verify the intrafractional tracking accuracy in infrared (IR) marker-based

37 hybrid dynamic tumour tracking irradiation ("IR Tracking") with the Vero4DRT.

38 Materials and Methods: The gimballed x-ray head tracks a moving target by predicting its

39 future position from displacements of IR markers in real-time. Ten lung cancer patients

40 who underwent IR Tracking were enrolled. The $95^{\text {th }}$ percentiles of intrafractional

41 mechanical $\left(i E_{M}^{95}\right)$, prediction $\left(i E_{P}^{95}\right)$, and overall targeting errors $\left(i E_{T}^{95}\right)$ were calculated

42 from orthogonal fluoroscopy images acquired during tracking irradiation and from the 43 synchronously acquired log files.

44 Results: Averaged intrafractional errors were (left-right, cranio-caudal [CC], 45 anterior-posterior $[\mathrm{AP}])=(0.1 \mathrm{~mm}, 0.4 \mathrm{~mm}, 0.1 \mathrm{~mm})$ for $i E_{M}^{95},(1.2 \mathrm{~mm}, 2.7 \mathrm{~mm}, 2.1 \mathrm{~mm})$

46 for $i E_{P}^{95}$, and $(1.3 \mathrm{~mm}, 2.4 \mathrm{~mm}, 1.4 \mathrm{~mm})$ for $i E_{T}^{95}$. By correcting systematic prediction

47 errors in the previous field, the $i E_{P}^{95}$ was reduced significantly, by an average of $0.4 \mathrm{~mm}$ 48 in the $\mathrm{CC}(p<0.05)$ and by $0.3 \mathrm{~mm}$ in the $\mathrm{AP}(p<0.01)$ directions.

49 Conclusions: Prediction errors were the primary cause of overall targeting errors, whereas

50 mechanical errors were negligible. Furthermore, improvement of the prediction accuracy

51 could be achieved by correcting systematic prediction errors in the previous field. 
Intrafractional accuracy of IR Tracking

\section{INTRODUCTION}

54 Respiratory motion is one of the factors causing uncertainties during beam delivery,

55 particularly for thoracic and abdominal tumours [1, 2]. In hypofractionated stereotactic

56 body radiotherapy for lung cancer patients, addition of a large margin to compensate for

57 respiratory motion increases the probability of complications [3]. Several techniques,

58 including forced shallow-breathing, breath-hold, respiratory gating, and dynamic tumour

59 tracking (DTT), have been proposed to reduce the uncertainties caused by respiratory

60 motion $[1,2]$. Of these methods, recent interest has focused on the DTT technique, which

61 can reposition the radiation beam dynamically in accordance with the target position. DTT

62 can minimise the internal uncertainties without a burden on the respiration of patients or

63 prolongation of treatment time.

We have developed an innovative four-dimensional (4D) image-guided

65 radiotherapy system, the Vero4DRT (MHI-TM2000; Mitsubishi Heavy Industries, Ltd.,

66 Japan, and BrainLAB, Feldkirchen, Germany) [4-10], and used its hybrid DTT irradiation

67 function [infrared (IR)-marker-based hybrid DTT irradiation ("IR Tracking")] clinically in

68 lung cancer patients since September 2011 [10]. In IR Tracking, the position of the target,

69 indicated by implanted fiducial markers, is calculated from external surrogate signals

70 through a pre-built prediction model (“4D model”), and the MV x-ray beam is delivered

71 with real-time monitoring [7, 8, 10-12]. Depuydt et al. showed that the performance of

72 Vero4DRT's DTT function was comparable with other clinical DTT systems in phantom

73 and patient simulation studies $[11,12]$. Our group also previously revealed that the

74 accuracy of the 4D model must be verified before treatment, and margins were required to 
Intrafractional accuracy of IR Tracking

75 compensate for the prediction error in a phantom study [7]; it was concluded that the

76 accuracy of the 4D model was affected by the baseline drift of respiratory motion [8]. Here,

77 we verified the intrafractional tracking accuracy of IR Tracking for lung cancer patients

78 using intrafractional monitoring images and the corresponding $\log$ files.

\section{MATERIALS AND METHODS}

\section{The Vero4DRT hybrid dynamic tumour tracking irradiation system}

82 Supplementary Figure 1 (Electronic Appendix) shows a schematic diagram of the

83 Vero4DRT system. The Vero4DRT has several unique components that facilitate DTT

84 irradiation: (1) a compact C-band 6-MV x-ray head with a gimbal mechanism, mounted on

85 an O-ring gantry. The gimballed x-ray head can swing itself in both the pan and tilt

86 directions, (2) gantry-mounted orthogonal $\mathrm{kV}$ x-ray imaging subsystems, consisting of two

87 sets of x-ray tubes and flat-panel detectors, with a spatial resolution of $0.2 \mathrm{~mm}$ at the

88 isocentre level, and (3) an extended version of the ExacTRAC system that enables real-time

89 motion monitoring and management for the DTT function $[7,8,11,12]$ with an IR camera

90 mounted on the ceiling of the treatment room.

91 Supplementary Figure 2 shows a schematic diagram of the IR Tracking procedure.

92 After patient positioning, a 4D model is created using synchronously monitored internal

93 target motion and an external surrogate signal. The detected target position $\left(P_{d}\right)$ is defined

94 as the tumour centre-of-mass calculated from the positions of the implanted fiducial

95 markers on the x-ray images. The relative shift amount between the tumour centre-of-mass

96 and centroid of the markers' polyhedron was determined at the end-exhalation phase in the 


\section{Intrafractional accuracy of IR Tracking}

97 planning computed tomography. The predicted target position $\left(P_{p}\right)$ is calculated from the

98 4D model, expressed by a quadratic equation involving two variables, the position and

99 velocity of the IR markers. The positions of the IR markers are predicted linearly from the 100 past motion to compensate for the DTT system delay [11]. Details of the prediction model 101 are described in the Supplementary Materials section. In this 4D-modelling phase, the 102 peak-to-peak amplitude of the detected target motion $(A)$ and the mean $(\mu)$ and standard 103 deviation $(S D)$ of the absolute $4 \mathrm{D}$-modelling error $\left(E_{4 D M}\right)$, defined as the absolute 104 difference between the $P_{p}$ and $P_{d}$, are calculated along each axis automatically. During 105 beam delivery, the future 3D target position is calculated from the displacements of the IR 106 markers using the 4D model, and then the corresponding tracking angle is transferred 107 continuously to the gimballed x-ray head. Additionally, circles with a user-defined radius 108 around the predicted positions of the fiducial markers (tolerance circles) are displayed on 109 the monitoring images as a benchmark in re-modelling. When the fiducial markers are 110 deviated systematically from the tolerance circles, re-modelling should be performed 111 during each treatment session (Fig. 1).

\section{Patient characteristics and treatment planning}

114 Ten lung cancer patients who underwent IR Tracking in an Institutional Review

115 Board-approved trial were included in the present study. Patient selection criteria were

116 based on our stereotactic body radiation therapy protocol and written informed consent for

117 the present study was obtained from each patient $[3,10]$. Three or more 1.5 -mm-diameter 118 gold markers (Olympus Co., Tokyo, Japan) were implanted around the lung tumour 


\section{Intrafractional accuracy of IR Tracking}

119 transbronchially 1-2 weeks before treatment planning. Table 1 shows the characteristics of

120 the patients and treatment planning. We performed a dry-run treatment session prior to

121 treatment planning to assess the characteristics of respirations and to identify

122 patient-specific planning target volume (PTV) margins [7, 9]. The median of $A$ was $2.8 \mathrm{~mm}$

123 in the left-right (LR), $15.8 \mathrm{~mm}$ in the cranio-caudal (CC), and $4.3 \mathrm{~mm}$ in the

124 anterior-posterior (AP) directions. The median of $\mu+2 S D$ of the $E_{4 D M}$ during the dry-run

125 treatment session $\left(E_{4 D M}^{\mu+2 S D}\right)$ was $0.6 \mathrm{~mm}$ in the $\mathrm{LR}, 1.9 \mathrm{~mm}$ in the $\mathrm{CC}$, and $0.7 \mathrm{~mm}$ in the AP

126 directions. Patient-specific PTV margins of 5.0-9.0 $\mathrm{mm}$ were added to the tumour along

127 each axis to compensate for intra- and interfractional uncertainties in IR Tracking [7, 9, 13].

128 Supplementary Figure 3 shows the definition of the patient-specific PTV margins. The

129 intra- and interfractional uncertainties were classified into systematic and random

130 components. The patient-specific PTV margins were then calculated for each axis using the

131 formula in Supplementary Figure 3. Prescribed doses of 48 or 56 Gy were specified to 132 isocentre in four fractions. Treatment plans included 6-8 non-coplanar fields, with a dose 133 rate of $500 \mathrm{MU} / \mathrm{min}$.

\section{Data acquisition during beam delivery}

136 During beam delivery, the target and fiducial markers were monitored using orthogonal $\mathrm{kV}$

137 x-ray imaging subsystems at $1 \mathrm{~Hz}$. The predicted target positions and tracking angles of the 138 gimballed x-ray head were recorded in $\log$ files at 60 and $200 \mathrm{~Hz}$, respectively. In total, 1399268 paired images ( 30 paired images per field) and corresponding log files were 140 acquired. 


\section{Intrafractional accuracy of IR Tracking}

\section{Verification of intrafractional tracking accuracy}

143 Intrafractional tracking accuracy was verified by the $P_{d}$ from the fluoroscopic images and 144 the corresponding $P_{p}$ and the tracked target position, calculated from the synchronously 145 acquired $\log$ files. Supplementary Figure 4 shows the geometric point of the tracked target 146 position at the depth of the $P_{d}\left(P_{t, d}\right)$. The tracked target position at the depth of the $P_{p}\left(P_{t, p}\right)$, 147 was calculated similarly. Intrafractional mechanical $\left(i E_{M}\right)$, prediction $\left(i E_{P}\right)$, and overall 148 targeting errors $\left(i E_{T}\right)$ were defined as the differences between $P_{t, p}$ and $P_{p}, P_{p}$ and $P_{d}$, and $P_{t, d}$ 149 and $P_{d}$, respectively. Details of the calculation process are described in the Supplementary 150 Materials section. The $95^{\text {th }}$ percentiles of the absolute $i E_{M}\left(i E_{M}^{95}\right), i E_{P}\left(i E_{P}^{95}\right)$, and $i E_{T}\left(i E_{T}^{95}\right)$ during

152 the treatment course were then calculated using the intrafractional monitoring images and 153 the corresponding log files. Pearson correlation coefficients were calculated to assess the

154 relationship between $E_{4 D M}^{\mu+2 S D}$ during the dry-run treatment session and $i E_{P}^{95}$ or $i E_{T}^{95}$ 155 during the treatment course. To further improve the prediction accuracy, the corrected $i E_{P}^{95}$ 156 was recalculated retrospectively by subtracting the systematic (i.e. signed overall mean) $i E_{P}$ 157 in the previous field excluding the first field after the 4D modelling. A paired $t$-test with a $158 \quad 0.05$ significance level was performed for statistical analysis.

\section{RESULTS}

161 Table 2 summarises $i E_{M}^{95}$, $i E_{P}^{95}, i E_{T}^{95}$, and corrected $i E_{P}^{95}$ for 10 lung cancer patients. 


\section{Intrafractional accuracy of IR Tracking}

162 Averaged intrafractional tracking errors were $(\mathrm{LR}, \mathrm{CC}, \mathrm{AP})=(0.1 \mathrm{~mm}, 0.4 \mathrm{~mm}, 0.1 \mathrm{~mm})$

163 for $i E_{M}^{95},(1.2 \mathrm{~mm}, 2.7 \mathrm{~mm}, 2.1 \mathrm{~mm})$ for $i E_{P}^{95}$, and $(1.3 \mathrm{~mm}, 2.4 \mathrm{~mm}, 1.4 \mathrm{~mm})$ for $i E_{T}^{95}$.

164 Additionally, a strong positive correlation was found between $E_{4 D M}^{\mu+2 S D}$ and $i E_{P}^{95}$ (LR, CC,

$165 \mathrm{AP})=(0.73[p=0.017], 0.82[p=0.003], 0.96[p=0.000])$ or $i E_{T}^{95}(\mathrm{LR}, \mathrm{CC}, \mathrm{AP})=(0.69$

$166[p=0.028], 0.77[p=0.010], 0.90[p=0.001])$. As shown in Table 2, $i E_{P}^{95}$ was the

167 primary cause of $i E_{T}^{95}$, while $i E_{M}^{95}$ was negligible. The $i E_{T}^{95}$ was fully covered by the

168 PTV margin, including the geometric variations between the tumour and fiducial markers.

169 Figure 2 (a) shows representative probability histograms in the positional error in the CC

170 direction for the first patient who underwent IR Tracking (Patient No. 1). $i E_{T}^{95}$ was

$1712.3 \mathrm{~mm}$ for this patient.

172 A maximum $i E_{T}^{95}$ of $4.1 \mathrm{~mm}$ was observed for Patient No. 7 in the CC direction.

173 This patient showed the largest difference between $E_{4 D M}^{\mu+2 S D}$ and $i E_{P}^{95}$ [LR, CC, and AP =

$1741.6,1.5$, and $1.6 \mathrm{~mm}$, respectively] due to a baseline drift during beam delivery. Meanwhile,

175 the averaged differences for the other patients were $0.3,0.6$, and $0.7 \mathrm{~mm}$ for the LR, CC,

176 and AP directions, respectively. By correcting the systematic prediction errors in the

177 previous field, however, $i E_{P}^{95}$ decreased, from 4.1 to $2.7 \mathrm{~mm}$, for this patient in the $\mathrm{CC}$

178 direction [Fig. 2 (b)]. The maximum reductions in $i E_{P}^{95}$ were observed in this patient (LR,

$179 \mathrm{CC}, \mathrm{AP})=(1.4 \mathrm{~mm}, 1.4 \mathrm{~mm}, 0.9 \mathrm{~mm})$. For the entire population, the corrected $i E_{P}^{95}$ was

180 improved significantly by an average of $0.4 \mathrm{~mm}$ in the $\mathrm{CC}(p<0.05)$ and by $0.3 \mathrm{~mm}$ in the $181 \operatorname{AP}(p<0.01)$ directions. 
Intrafractional accuracy of IR Tracking

\section{DISCUSSION}

184 The Vero4DRT tracks a moving target in real-time using the orthogonal gimballed x-ray

185 head. In the present study, we established a verification methodology for the intrafractional

186 mechanical, prediction, and overall targeting accuracy in each axis during the treatment

187 course. The 3D coordinates of the intrafractional tracked target position were calculated

188 based on the MV x-ray beam orientation using intrafractional monitoring images and the 189 corresponding log files.

190 We verified the intrafractional tracking accuracy for 10 lung cancer patients who

191 underwent IR Tracking with real-time monitoring. Vero4DRT users can monitor the moving 192 target, fiducial markers, and tolerance circles with its predicted position using orthogonal 193 kV x-ray imaging subsystems during beam delivery. At our institution, the radius of the 194 tolerance circles is set to $3 \mathrm{~mm}$, and the 4D model is re-modelled when the monitored 195 fiducial markers' positions are displaced systematically from the tolerance circles due to 196 baseline drift (Fig. 1). By re-modelling the 4D model, while an $i E_{T}^{95}$ of less than $3 \mathrm{~mm}$ 197 was achieved for nine patients (90\%), one patient (Patient No. 7) showed a large $i E_{T}^{95}$ of 198 greater than $3 \mathrm{~mm}$. The 4D model was updated once during the treatment session for

199 Patient No. 7. However, this patient required additional re-modelling. In IR Tracking, the 200 predominant cause of overall targeting errors was prediction errors. The position and 201 velocity of IR markers involved in the 4D model were predicted linearly from past IR 202 marker motion [8]. Thus, prediction uncertainty of the peak position sometimes 203 overestimated the predicted position of the IR marker and the 4D model enforced a large 


\section{Intrafractional accuracy of IR Tracking}

204 amplitude of respiration motion (Supplementary Figure 5). In this case, the mechanical

205 response delay of the gimballed x-ray head reduced the impact of the prediction error on

206 the overall targeting error. Thus, the overall targeting errors were sometimes smaller than

207 the prediction errors. Additionally, there were strong correlations between $E_{4 D M}^{\mu+2 S D}$ and

$208 i E_{P}^{95}$ or $i E_{T}^{95}$, indicating that intrafractional prediction or overall targeting errors during

209 the treatment course could be estimated from 4D modelling errors during the dry-run

210 treatment session. The $i E_{T}^{95}$ was fully covered by the PTV margin, including a geometric

211 variation between the tumour and fiducial markers of $2.5 \mathrm{~mm}$ (Tables 1 and 2). When

212 calculating the PTV margin in IR Tracking, the intra- and interfractional uncertainties

213 should be considered (Supplementary Figure 3). However, the present recipe of the

214 patient-specific PTV margin was tentative so as to perform IR Tracking safely. Therefore,

215 further investigations will be needed to determine the PTV margin size appropriate for IR

216 Tracking [9].

217 The CyberKnife Robotic Radiosurgery System with the integrated Synchrony

218 Respiratory Tracking System (Accuray, Sunnyvale, CA) substantially reduces the

219 geometric error caused by respiratory motion $[14,15]$. In the present study, $E_{4 D M}^{\mu+2 S D}$ was

220 comparable with results of the Synchrony system. However, the correlation between the

221 internal target positions and external surrogates can change in the presence of baseline drift,

222 reducing the accuracy of the prediction model $[8,16]$. The Synchrony system periodically

223 updates the prediction model using the intrafractional monitoring images. Updating the 4D

224 model in real-time may also improve the prediction accuracy because the internal/external 


\section{Intrafractional accuracy of IR Tracking}

225 correlation change or baseline drift in respiration will be corrected. Meanwhile, this is

226 difficult regarding image processing time and minimum interval of the $\mathrm{x}$-ray acquisition

227 during beam delivery. The 4D model in IR Tracking includes the parameters of position and

228 velocity of the IR markers. To update the $4 \mathrm{D}$ model, these parameters must be changed.

229 Thus, a shorter monitoring interval would be necessary. In clinical practice, we re-modelled

230 the 4D model at least once during treatment to minimise intrafractional uncertainties due to

231 internal/external correlation change or baseline drift in respiration. However, re-modelling

232 required additional exposures that were 8.3-16.7 times higher than intrafractional

233 monitoring $[4,12]$. Also, $x$-ray image-based DTT, another DTT approach with Vero4DRT

234 [6], would not be an alternative strategy in terms of the difficulty of real-time detection and

235 excessive imaging doses. In the current study, the overall mean errors of $i E_{P}$ were

236 calculated from around 30 paired images retrieved in the previous field using the

237 monitoring function for the intrafractional tracking accuracy verification. Because the

238 systematic prediction errors resulting from the baseline drift of respiration were reduced by

239 subtracting the overall mean errors of $i E_{P}$ in the previous field, $i E_{P}^{95}$ decreased

240 significantly in the $\mathrm{CC}$ and AP directions using the monitoring images during beam delivery.

241 In the current study, we used all monitoring images to calculate the systematic prediction

242 errors because $i E_{P}$ varied according to the respiratory phase. However, a triggered x-ray

243 acquisition based on the respiratory phase would also reduce $i E_{P}^{95}$ using a small number

244 of monitoring images because the systematic prediction errors could be corrected by the

245 averaged $i E_{P}$ at the end-expiratory and end-inspiratory phases. 
Intrafractional accuracy of IR Tracking

248 We demonstrated that IR Tracking reduced the impact of respiratory motion substantially.

249 The prediction error was the primary cause of the overall targeting error, while the 250 mechanical error was negligible. The PTV margin fully covered the intrafractional overall 251 targeting errors. The 4D modelling errors during a dry-run treatment session were a good 252 indicator of the prediction and overall targeting errors during the treatment course. 253 Additionally, further improvement in prediction accuracy was achieved by correcting the 254 systematic prediction error in the previous field.

257 This research was sponsored in part by Mitsubishi Heavy Industries, Ltd., Japan. Takashi 258 Mizowaki, Masaki Kokubo, and Masahiro Hiraoka have consultancy agreements with 259 Mitsubishi Heavy Industries, Ltd.

\section{ACKNOWLEDGEMENTS}

262 This research was supported by the Japan Society for the Promotion of Science (JSPS) 263 through its "Funding Program for World-Leading Innovative R\&D on Science and 264 Technology (FIRST Program).” 
Intrafractional accuracy of IR Tracking

\section{REFERENCES}

267 [1] Keall P J, Mageras G S, Balter J M, et al. The management of respiratory motion in 268 radiation oncology report of AAPM task group 76. Med Phys 2006;33:3874-900.

269 [2] Korreman S S. Motion in radiotherapy: photon therapy. Phys Med Biol $270 \quad$ 2012;57:R161-91.

271 [3] Matsuo Y, Shibuya K, Nakamura M, et al. Dose-volume metrics associated with 272 radiation pneumonitis after stereotactic body radiation therapy for lung cancer. Int $\mathbf{J}$ 273 Radiat Oncol Biol Phys 2012;83:e545-9.

274 [4] Kamino Y, Takayama K, Kokubo M, et al. Development of a four-dimensional 275 image-guided radiotherapy system with a gimbaled X-ray head. Int J Radiat Oncol Biol 276 Phys 2006;66:271-8.

277 [5] Takayama K, Mizowaki T, Kokubo M, et al. Initial validations for pursuing irradiation 278 using a gimbals tracking system. Radiother Oncol 2009;93:45-9.

279 [6] Mukumoto N, Nakamura M, Sawada A, et al. Positional accuracy of novel 280 x-ray-image-based dynamic tumor-tracking irradiation using a gimbaled MV x-ray 281 head of a Vero4DRT (MHI-TM2000). Med Phys 2012;39:6287-96.

282 [7] Mukumoto N, Nakamura M, Sawada A, et al. Accuracy verification of infrared 283 marker-based dynamic tumor-tracking irradiation using the gimbaled x-ray head of the Vero4DRT (MHI-TM2000). Med Phys 2013;40:041706-1-9.

285 [8] Akimoto M, Nakamura M, Mukumoto N, et al. Predictive uncertainty in infrared 286 marker-based dynamic tumor tracking with Vero4DRT. Med Phys 2013;40:091705 $287-1-8$. 
Intrafractional accuracy of IR Tracking

288 [9] Nakamura M, Mukumoto N, Ueki N, et al. Estimation of a tracking margin in 289 surrogate signal-based dynamic tumor tracking irradiation with Vero4DRT. Int J Radiat $290 \quad$ Oncol Biol Phys 2012;84:S851-2.

291 [10]Matsuo Y, Sawada A, Ueki N, et al. An initial experience of dynamic tumor tracking 292 irradiation with real-time monitoring using Vero4DRT (MHI-TM2000). Radiother $293 \quad$ Oncol 2012;103:S64.

294 [11]Depuydt T, Verellen D, Haas O, et al. Geometric accuracy of a novel gimbals based 295 radiation therapy tumor tracking system. Radiother Oncol 2011;98:365-72.

296 [12]Depuydt T, Poelsa K, Verellen D, et al. Initial assessment of tumor tracking with a 297 gimbaled linac system in clinical circumstances: A patient simulation study. Radiother $298 \quad$ Oncol 2013;106:236-40.

299 [13]Ueki N, Matsuo Y, Nakamura M, et al. Intra- and interfractional variations in geometric arrangement between lung tumours and implanted markers. Radiother Oncol 2013, in

302 [14]Hoogeman M, Prévost J B, Nuyttens J, et al. Clinical accuracy of the respiratory tumor 303 tracking system of the cyberknife: assessment by analysis of log files. Int $\mathbf{J}$ Radiat $304 \quad$ Oncol Biol Phys 2009;74:297-303.

305 [15]Pepin E W, Wu H, Zhang Y, et al. Correlation and prediction uncertainties in the 306 cyberknife synchrony respiratory tracking system. Med Phys 2011;38:4036-44.

307 [16]Malinowski K, McAvoy $\mathrm{T} \mathrm{J}$, George $\mathrm{R}$, et al. Incidence of changes in 308 respiration-induced tumor motion and its relationship with respiratory surrogates 309 during individual treatment fractions. Int J Radiat Oncol Biol Phys 2012;82:1665-73. 
Intrafractional accuracy of IR Tracking

312 Figure 1. Screen shot of the Vero4DRT during infrared (IR)-marker-based DTT irradiation

313 ("IR Tracking"). Monitored fiducial markers' positions were located outside of the

314 "Tolerance circle" displayed around the predicted fiducial markers' positions due to the 315 baseline drift of respiration.

316

317 Figure 2. Probability histograms of positional errors in the cranio-caudal (CC) direction (a) 318 for the first patient who underwent IR Tracking (Patient No. 1) and (b) for the most 319 improved patient with intrafractional prediction error $\left(i E_{P}\right)$ correction (Patient No. 7). The 320 Vero4DRT reduced the motion blurring effect caused by respiration. 


\section{TABLES}

Table 1. Characteristics of the patients and treatment planning.

\begin{tabular}{|c|c|c|c|c|c|c|c|c|c|c|c|c|c|c|c|c|}
\hline \multirow{2}{*}{$\begin{array}{c}\text { Patient } \\
\text { no. }\end{array}$} & \multirow{2}{*}{$\begin{array}{l}\text { Age } \\
\text { (y.o.) }\end{array}$} & \multirow{2}{*}{ Sex } & \multirow{2}{*}{$\begin{array}{l}\text { Tumour } \\
\text { stage }\end{array}$} & \multirow{2}{*}{$\begin{array}{l}\text { Tumour } \\
\text { side }\end{array}$} & \multirow{2}{*}{$\begin{array}{l}\text { Tumour } \\
\text { location }\end{array}$} & \multicolumn{3}{|c|}{$A[\mathrm{~mm}]$} & \multicolumn{3}{|c|}{$E_{4 D M}^{\mu+2 S D} \quad[\mathrm{~mm}]$} & \multirow{2}{*}{$\begin{array}{l}\text { GTV } \\
{[\mathrm{cc}]}\end{array}$} & \multicolumn{3}{|c|}{ PTV margin $[\mathrm{mm}]$} & \multirow{2}{*}{$\begin{array}{l}\text { PTV } \\
{[\mathrm{cc}]}\end{array}$} \\
\hline & & & & & & LR & $\mathrm{CC}$ & AP & LR & $\mathrm{CC}$ & AP & & LR & $\mathrm{CC}$ & AP & \\
\hline 2 & 82 & M & T1a & Rt & S9 & 4.6 & 19.8 & 4.4 & 1.6 & 3.3 & 0.5 & 11.0 & 5.0 & 8.0 & 5.0 & 34.6 \\
\hline 3 & 86 & $\mathrm{~F}$ & $\mathrm{~T} 1 \mathrm{~b}$ & Rt & S9 & 2.2 & 26.0 & 5.3 & 0.2 & 1.6 & 0.8 & 12.4 & 5.0 & 8.0 & 5.0 & 38.0 \\
\hline 4 & 84 & M & $\mathrm{T} 1 \mathrm{~b}$ & Rt & S6 & 0.9 & 11.9 & 3.1 & 0.3 & 1.3 & 0.7 & 17.8 & 5.0 & 7.0 & 5.0 & 50.1 \\
\hline 5 & 71 & M & $\mathrm{T} 1 \mathrm{~b}$ & Rt & S5 & 7.4 & 3.4 & 5.1 & 1.3 & 1.0 & 0.7 & 12.5 & 5.0 & 5.0 & 5.0 & 33.6 \\
\hline 6 & 87 & M & $\mathrm{T} 1 \mathrm{~b}$ & $\mathrm{Lt}$ & $\mathrm{S} 8$ & 2.9 & 29.6 & 15.5 & 0.6 & 2.2 & 3.9 & 20.6 & 5.0 & 8.0 & 8.0 & 64.1 \\
\hline 7 & 61 & M & $\mathrm{T} 2 \mathrm{a}$ & Rt & S10 & 1.4 & 8.8 & 4.0 & 0.5 & 2.6 & 0.7 & 31.9 & 5.0 & 6.5 & 5.0 & 74.2 \\
\hline 8 & 85 & M & $\mathrm{T} 1 \mathrm{a}$ & $\mathrm{Lt}$ & S9 & 5.8 & 30.6 & 8.7 & 2.1 & 3.4 & 2.9 & 8.9 & 5.0 & 9.0 & 6.0 & 33.4 \\
\hline 9 & 68 & M & Metastasis & $\mathrm{Lt}$ & S8 & 2.7 & 9.9 & 2.3 & 0.2 & 0.9 & 0.4 & 2.3 & 5.0 & 5.0 & 5.0 & 10.4 \\
\hline 10 & 60 & M & Metastasis & $\mathrm{Rt}$ & S9 & 3.1 & 16.7 & 4.1 & 0.9 & 2.2 & 1.6 & 13.3 & 5.0 & 7.0 & 5.0 & 38.9 \\
\hline
\end{tabular}

Abbreviations: $A=$ peak-to-peak amplitude of respiration, $E_{4 D M}^{\mu+2 S D}=$ mean plus two standard deviations of the absolute $4 \mathrm{D}-\mathrm{modelling}$ error during a dry-run treatment session, GTV=gross tumour volume, PTV=planning target volume, LR=left-right, CC=cranio-caudal, $\mathrm{AP}=$ anterior-posterior, $\mathrm{F}=$ Female, $\mathrm{M}=$ Male, $\mathrm{Rt}=$ Right lobe, $\mathrm{Lt}=$ Left lobe, $\mathrm{S}=$ pulmonary segment. 
Table 2. $i E_{M}^{95}, i E_{P}^{95}, i E_{T}^{95}$, and corrected $i E_{P}^{95}$.

\begin{tabular}{ccccccccccccc}
\hline $\begin{array}{c}\text { Patient } \\
\text { no. }\end{array}$ & \multicolumn{3}{c}{$i E_{M}^{95}[\mathrm{~mm}]$} & \multicolumn{3}{c}{$i E_{P}^{95}[\mathrm{~mm}]$} & \multicolumn{3}{c}{$i E_{T}^{95}[\mathrm{~mm}]$} & \multicolumn{2}{c}{ Corrected $i E_{P}^{95}$} & {$[\mathrm{~mm}]$} \\
& LR & CC & AP & LR & CC & AP & LR & CC & AP & LR & CC & AP \\
\hline 1 & 0.1 & 0.3 & 0.1 & 0.6 & 2.7 & 1.3 & 0.9 & 2.3 & 1.0 & 0.6 & 1.9 & 1.2 \\
2 & 0.3 & 0.7 & 0.2 & 1.4 & 3.4 & 0.8 & 1.3 & 3.0 & 0.7 & 1.3 & 3.1 & 0.6 \\
3 & 0.1 & 0.4 & 0.1 & 0.7 & 2.5 & 1.5 & 0.8 & 2.2 & 1.0 & 0.7 & 2.7 & 1.3 \\
4 & 0.1 & 0.4 & 0.1 & 0.8 & 2.0 & 1.5 & 0.8 & 1.7 & 1.0 & 0.8 & 1.7 & 1.4 \\
5 & 0.1 & 0.1 & 0.1 & 1.8 & 1.5 & 1.6 & 1.1 & 1.5 & 1.2 & 1.5 & 1.5 & 1.6 \\
6 & 0.1 & 0.3 & 0.1 & 1.0 & 2.5 & 5.0 & 1.9 & 2.3 & 2.9 & 0.9 & 1.8 & 4.3 \\
7 & 0.2 & 0.8 & 0.2 & 2.1 & 4.1 & 2.3 & 1.4 & 4.1 & 1.6 & 0.7 & 2.7 & 1.4 \\
8 & 0.2 & 0.4 & 0.1 & 2.1 & 3.2 & 3.4 & 2.2 & 2.8 & 1.6 & 2.5 & 3.2 & 3.2 \\
9 & 0.1 & 0.3 & 0.1 & 0.5 & 1.6 & 1.4 & 0.8 & 1.4 & 0.9 & 0.4 & 1.3 & 1.1 \\
10 & 0.1 & 0.3 & 0.1 & 1.1 & 3.0 & 2.0 & 1.3 & 2.9 & 1.6 & 1.0 & 2.7 & 1.8 \\
\hline Average & 0.1 & 0.4 & 0.1 & 1.2 & 2.7 & 2.1 & 1.3 & 2.4 & 1.4 & 1.0 & 2.3 & 1.8 \\
\hline
\end{tabular}

Abbreviations: $i E_{M}^{95}=95^{\text {th }}$ percentiles of the absolute intrafractional mechanical error, $i E_{P}^{95}=95^{\text {th }}$ percentiles of the absolute intrafractional prediction error, $i E_{T}^{95}=95^{\text {th }}$ percentiles of the absolute intrafractional overall targeting error, LR=left-right, $\mathrm{CC}=$ cranio-caudal, $\mathrm{AP}=$ anterior-posterior. 
Click here to download high resolution image
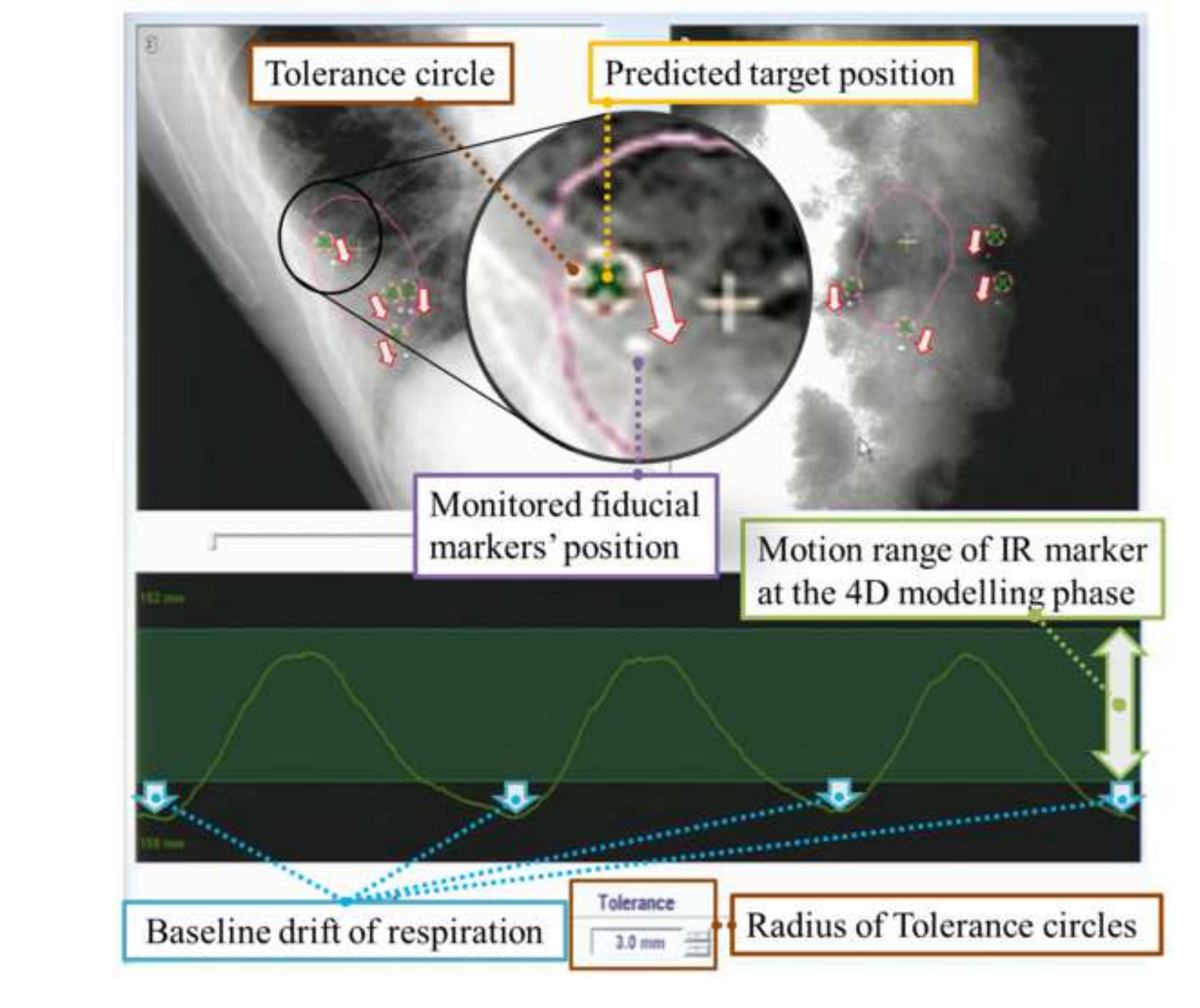

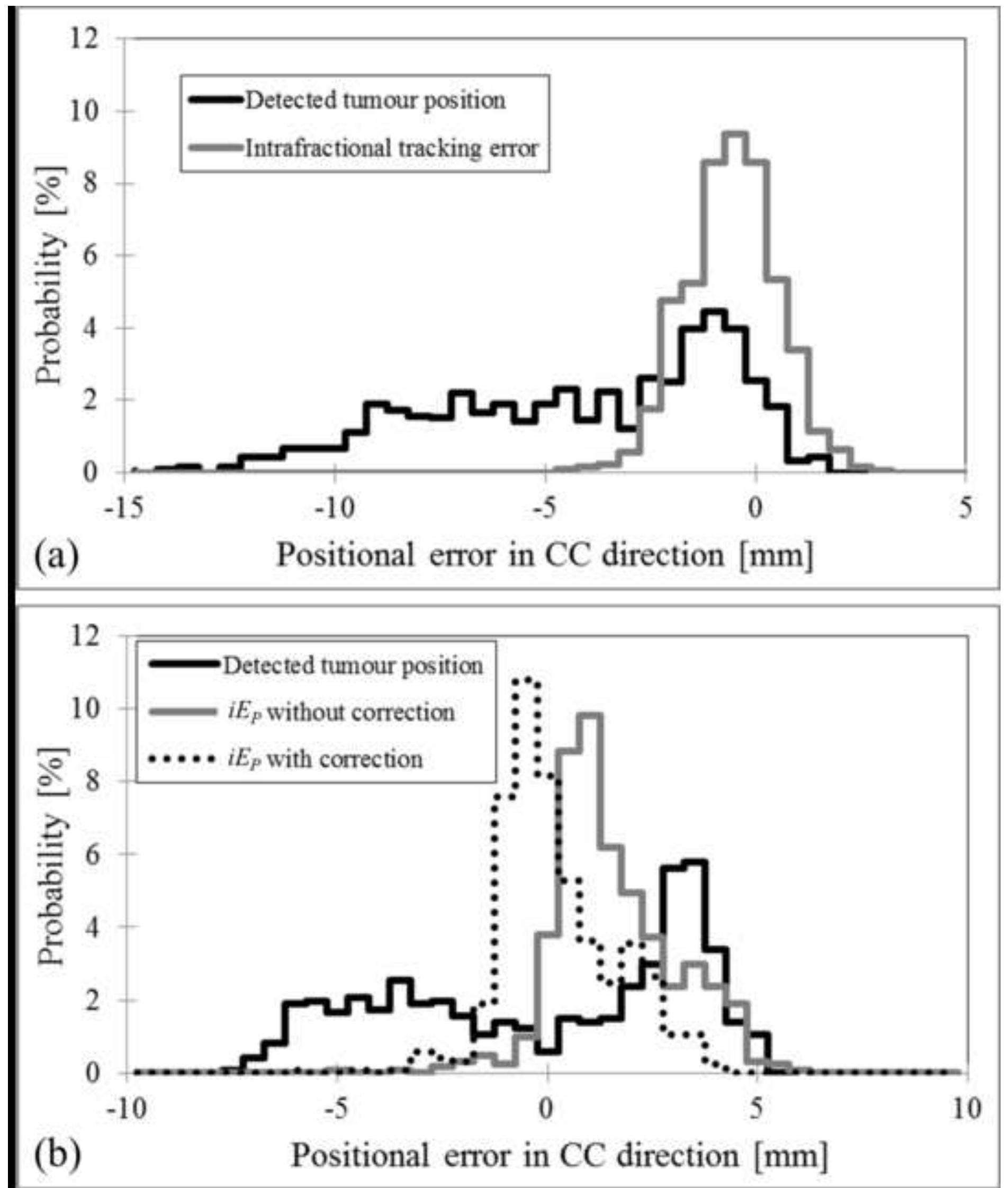
(b)
Positional error in CC direction [mm] 
Mukumoto et al. Intrafractional accuracy of IR Tracking

\section{$1 \quad$ Prediction model of the Vero4DRT}

2 Before irradiation, a prediction model ("4D model”) was created. Infrared (IR) marker

3 displacements and the implanted fiducial markers' motions were monitored for 20-40 s

4 using the IR camera of the ExacTRAC system every $16.7 \mathrm{~ms}$ and the orthogonal $\mathrm{kV}$ x-ray

5 imaging subsystems every 80 or $160 \mathrm{~ms}$, respectively. The frame rate of x-ray monitoring

6 changed automatically depending on IR marker velocity.

After monitoring, two target positions are determined: the detected target position

$8 \quad\left(P_{d}\right)$ and the predicted target position $\left(P_{p}\right)$. The $P_{d}$ is defined as the tumour centre-of-mass

9 calculated from the positions of the implanted fiducial markers on the x-ray images. The

10 relative shift amount between the tumour centre-of-mass and centroid of the markers'

11 polyhedron was determined at the end-exhalation phase in the planning computed

12 tomography. The positions of the implanted fiducial markers were detected automatically

13 based on the intensity ratios of the fiducial markers to their surroundings with an accuracy

14 of $0.2 \mathrm{~mm}$. The $P_{p}$ is calculated from the predicted position and velocity of IR markers

15 using the 4D model, expressed as follows:

$16 P_{p}=\left(\begin{array}{l}x_{p} \\ y_{p} \\ z_{p}\end{array}\right)=\frac{1}{n}\left(\begin{array}{l}\sum_{i=1}^{n}\left(a_{x, i} s_{i}^{2}+b_{x, i} s_{i}+c_{x, i}+d_{x, i} v_{i}^{2}+e_{x, i} v_{i}\right) \\ \sum_{i=1}^{n}\left(a_{y, i} s_{i}^{2}+b_{y, i} s_{i}+c_{y, i}+d_{y, i} v_{i}^{2}+e_{y, i} v_{i}\right) \\ \sum_{i=1}^{n}\left(a_{z, i} s_{i}^{2}+b_{z, i} s_{i}+c_{z, i}+d_{z, i} v_{i}^{2}+e_{z, i} v_{i}\right)\end{array}\right)$ (equation1),

17 where $x_{p}, y_{p}$, and $z_{p}$ are the predicted target positions in the left-right, cranio-caudal, and anterior-posterior directions, $n$ is the number of IR markers, and $s$ and $v$ are the predicted position and velocity of each IR marker in the anterior-posterior direction. The positions of 
Mukumoto et al. Intrafractional accuracy of IR Tracking

20 the IR markers are predicted from the past motion to compensate for DTT system delay.

21 Parameters of the 4D model $(a, b, c, d$, and $e$ ) were optimised using a least-squares

22 algorithm so that residual errors between the $P_{p}$ and $P_{d}$ were minimised.

23 During beam delivery, the future 3D target position is predicted from the

24 displacements of the IR markers using the 4D model, and then the corresponding tracking

25 angle is transferred continuously to the gimballed x-ray head.

26 
Mukumoto et al. Intrafractional accuracy of IR Tracking

\section{Tracked target position calculated from the tracking angle of the gimballed $x$-ray head}

Intrafractional tracking accuracy was assessed by the detected target position $\left(P_{d}\right)$ from the fluoroscopic images and the corresponding predicted target position $\left(P_{p}\right)$ and the tracked target position, calculated from the synchronously acquired log files. The tracked target position was derived from an intersection of a tracking orientation of the gimballed x-ray head with a tracked tumour plane. The tracked tumour plane was defined as the perpendicular plane to the gimbal angle of $0^{\circ}$ for each port at the depth of the moving tumour. The tracked target position, based on $P_{d}\left(P_{t, d}\right)$, was calculated in the following three steps:

(1) Conversion of $P_{d}$ from room to gantry-ring coordinates:

(1)

$$
\left(\begin{array}{l}
u_{d} \\
v_{d} \\
w_{d}
\end{array}\right)=\left(\begin{array}{ccc}
\cos G \cos R & -\cos G \sin R & -\sin G \\
-\sin R & -\cos R & 0 \\
\sin G \cos R & -\sin G \sin R & \cos G
\end{array}\right) \cdot\left(\begin{array}{l}
x_{d} \\
y_{d} \\
z_{d}
\end{array}\right) \text { (equation 2), }
$$

where $x_{d}, y_{d}$, and $z_{d}$ are the detected target positions along the LR, the CC, and the AP directions in room coordinates, and $G$ and $R$ are the gantry and ring angle, and $u_{d}, v_{d}$, and $w_{d}$ (units: $\mathrm{mm}$ ) are the detected target positions in gantry-ring coordinates corresponding to $x_{d}, y_{d}$, and $z_{d}$

(2) Calculation of $P_{t, d}$ at the depth of $P_{d}$ in gantry-ring coordinates

$$
\left.\left(\begin{array}{c}
u_{t, d} \\
v_{t, d} \\
w_{t, d}
\end{array}\right)=\left(\begin{array}{c}
\left(960-w_{d}\right) \tan \theta_{P} \\
\left(960-w_{d}\right) \tan \theta_{T} \\
w_{d}
\end{array}\right) \text { (equation } 3\right)
$$

where $u_{t, d}, v_{t, d}$, and $w_{t, d}$ (units: $\mathrm{mm}$ ) are the tracked target positions in gantry-ring 
Mukumoto et al. Intrafractional accuracy of IR Tracking

45

46

47

48

coordinates at the depth of the detected target position $\left(w_{t, d}\right) . \theta_{P}$ and $\theta_{T}$ are the pan and tilt angle of the gimballed x-ray head, and $960 \mathrm{~mm}$ is the distance from the rotation centre of the gimballed x-ray head to the isocentre.

(3) Conversion of $P_{t, d}$ from gantry-ring to room coordinates:

$$
\left(\begin{array}{l}
x_{t, d} \\
y_{t, d} \\
z_{t, d}
\end{array}\right)=\left(\begin{array}{ccc}
\cos G \cos R & -\sin R & \sin G \cos R \\
-\cos G \sin R & -\cos R & -\sin G \sin R \\
-\sin G & 0 & \cos G
\end{array}\right) \cdot\left(\begin{array}{c}
u_{t, d} \\
v_{t, d} \\
w_{t, d}
\end{array}\right) \text { (equation 4), }
$$

where $x_{t, d}, y_{t, d}$, and $z_{t, d}$ (units: $\mathrm{mm}$ ) are the tracked target positions in room coordinates. The tracked target position, based on $P_{p}\left(P_{t, p}\right)$, at the depth of the predicted target position $\left(w_{p}\right)$ was calculated similarly.

Intrafractional mechanical $\left(i E_{M}\right)$, prediction $\left(i E_{P}\right)$, and overall targeting errors $\left(i E_{T}\right)$ were defined as follows:

$$
\begin{aligned}
& i E_{M}=\left(\begin{array}{l}
x_{t, p} \\
y_{t, p} \\
z_{t, p}
\end{array}\right)-\left(\begin{array}{l}
x_{p} \\
y_{p} \\
z_{p}
\end{array}\right) \text { (equation 5), } \\
& i E_{P}=\left(\begin{array}{l}
x_{p} \\
y_{p} \\
z_{p}
\end{array}\right)-\left(\begin{array}{l}
x_{d} \\
y_{d} \\
z_{d}
\end{array}\right) \text { (equation 6), } \\
& i E_{T}=\left(\begin{array}{l}
x_{t, d} \\
y_{t, d} \\
z_{t, d}
\end{array}\right)-\left(\begin{array}{l}
x_{d} \\
y_{d} \\
z_{d}
\end{array}\right) \text { (equation 7), }
\end{aligned}
$$

where $x_{t, p}, y_{t, p}$, and $z_{t, p}$ (units: $\mathrm{mm}$ ) are the tracked target positions at the depth of the $P_{p}$ used for the verification of the mechanical error of the gimballed x-ray head against the 
Mukumoto et al. Intrafractional accuracy of IR Tracking

60 predicted target positions, and $x_{p}, y_{p}$, and $z_{p}$ (units: $\mathrm{mm}$ ) are the predicted target positions

61 used as the tracking commands to the gimballed x-ray head, and $x_{d}, y_{d}$, and $z_{d}$ (units: mm)

62 are the detected target positions, and $x_{t, d}, y_{t, d}$, and $z_{t, d}$ (units: mm) are the tracked target

63 positions at the depth of the $P_{d}$ used for the verification of the overall targeting error of the 64 gimballed $\mathrm{x}$-ray head against the moving tumour. 


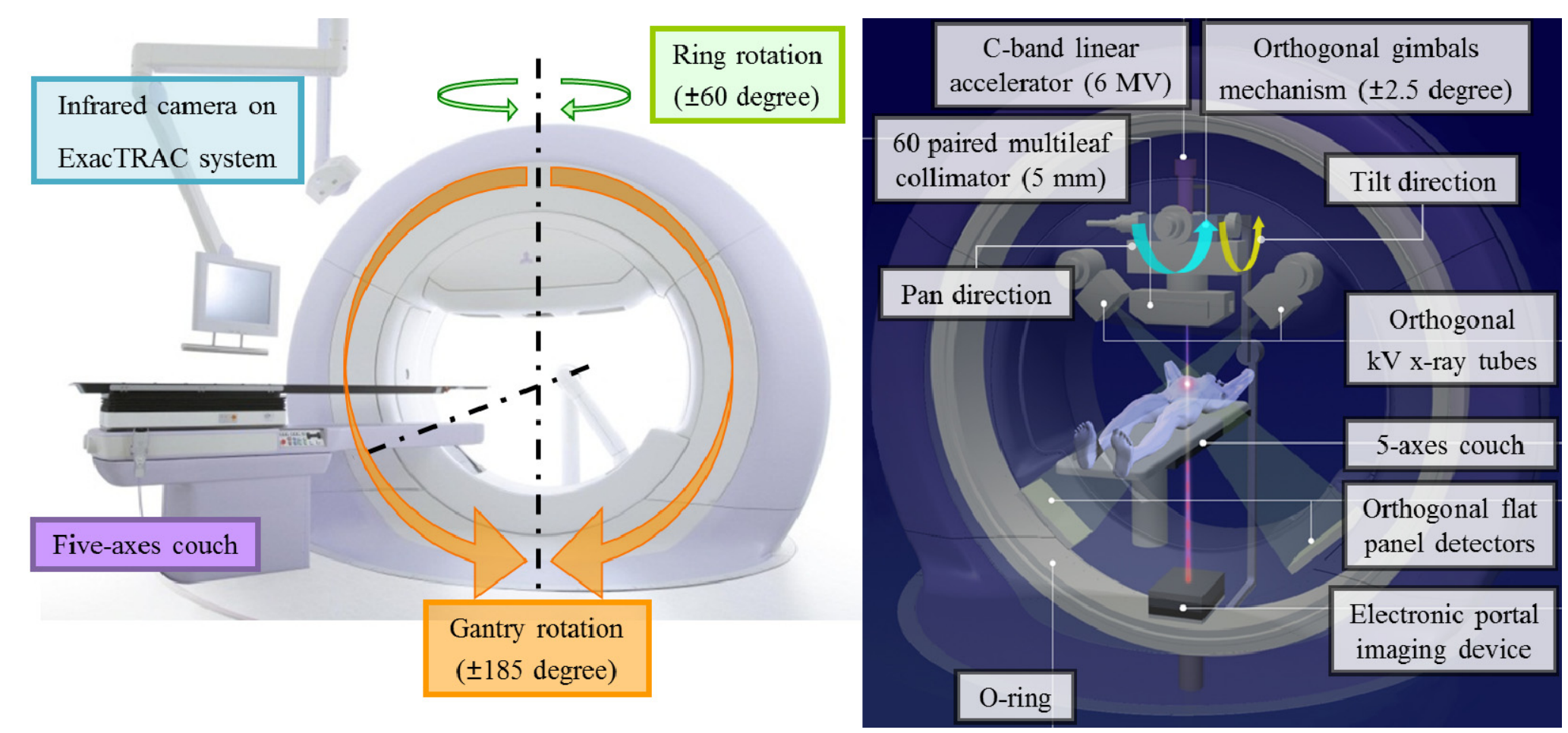

65

66 Supplementary Figure 1. Schematic diagram of the Vero4DRT system. 
Mukumoto et al. Intrafractional accuracy of IR Tracking
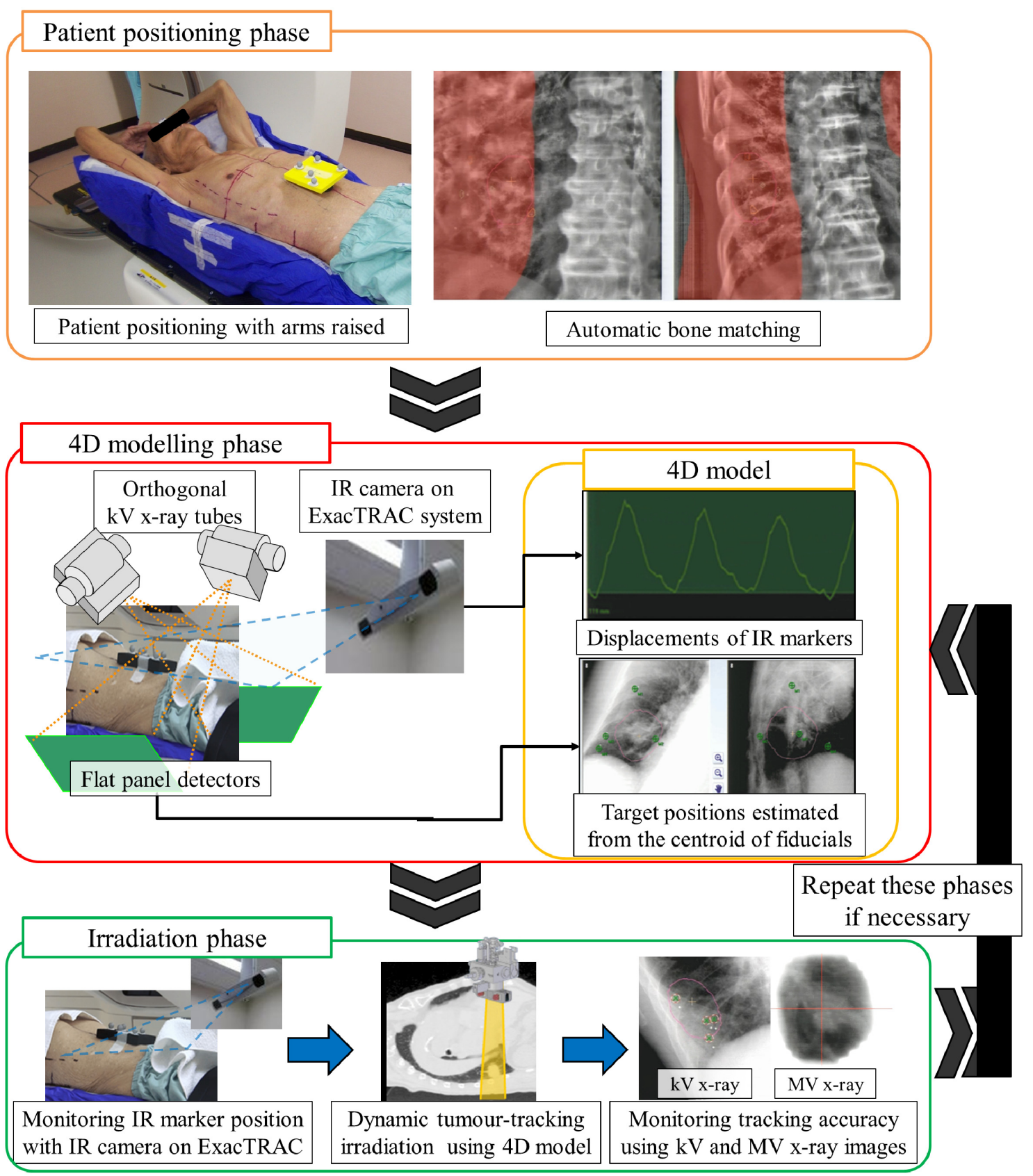

68 Supplementary Figure 2. Infrared (IR) marker-based hybrid dynamic tumour tracking irradiation (“IR Tracking") procedure. 


\section{Patient-specific PTV margin}

(1) Interfractional error

- Geometric uncertainty between $\operatorname{Marker}\left(\mathrm{M}_{\mathrm{n}}\right)$ and Target $\left(\mathrm{T}_{\mathrm{n}}\right)$

$-2.5 \mathrm{~mm}$

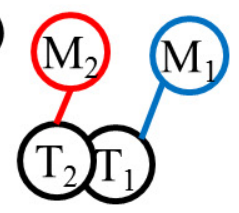

(2) Intrafractional error (Systematic)

- Baseline drift of respiration - $10 \%$ of peak-to-peak Amplitude
(3) Intrafractional error (Random)

- 4D modelling error

- Mean + 2SD of absolute 4D modelling error

(4) Intrafractional error (Random)

- Mechanical error

$-95^{\text {th }}$ percentiles of mechanical error

\section{$P T V$ margin $[\mathrm{mm}]=(1)+(2)+\sqrt{(3)^{2}+(4)^{2}}$ Minimum size of PTV margin was set to $5 \mathrm{~mm}$}

71 Supplementary Figure 3. Definition of the patient-specific planning target volume (PTV) margin. 


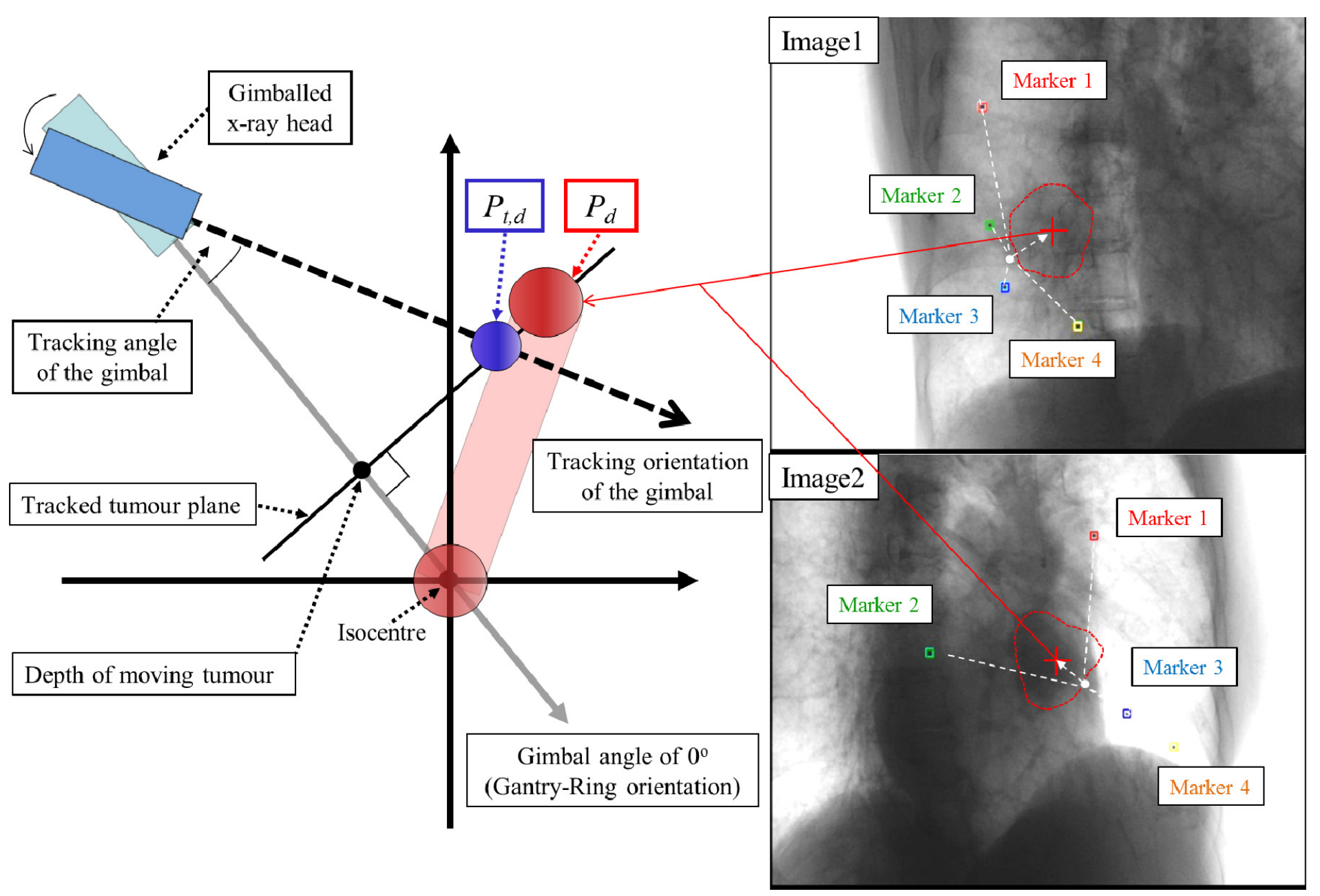

73 Supplementary Figure 4. The geometric point of the tracked target position $\left(P_{t, d}\right)$ based on the detected target position $\left(P_{d}\right)$

74 calculated from orthogonal fluoroscopic images and synchronously acquired log files. 


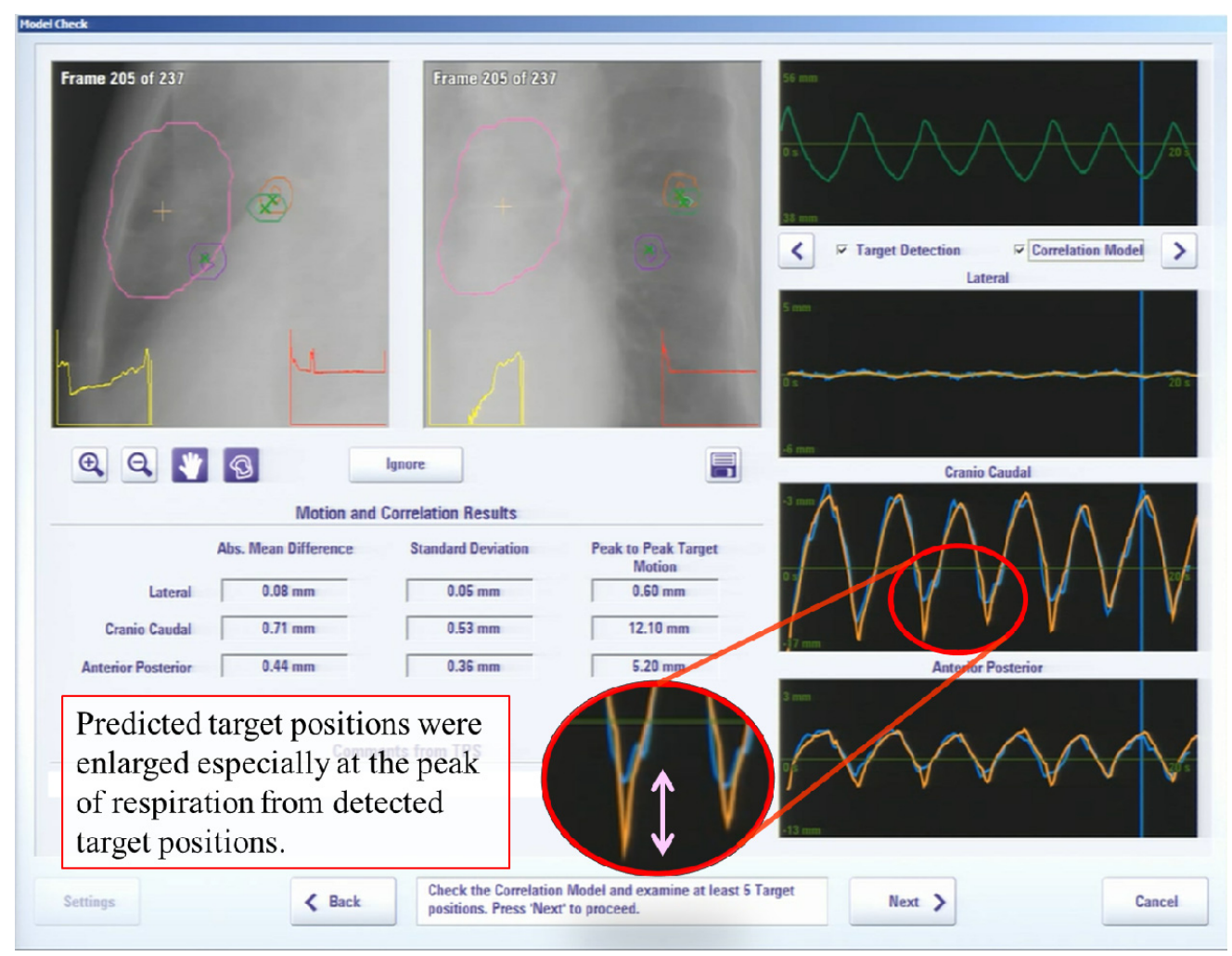

76 Supplementary Figure 5. Screen shot of the Vero4DRT system during creation of the prediction model ("4D model"). The

77 right four groups of waves, from top to bottom, show variations in the infrared (IR) markers' positions in the

78 anterior-posterior direction and the target positions in the lateral, craniocaudal, and anterior-posterior directions, respectively.

79 In the graphs of the target position, dark-coloured waves show the detected target position and light-coloured waves show the

80 predicted target position. 